\title{
Texture of GaAs Nanoparticles Deposited by Pulsed Laser Ablation in Different Atmospheres
}

\author{
P. Dubček, ${ }^{1}$ B. Pivac, ${ }^{1}$ S. Milošević, ${ }^{2}$ N. Krstulović, ${ }^{2}$ Z. Kregar, ${ }^{2}$ and S. Bernstorff ${ }^{3}$ \\ ${ }^{1}$ R. Bošković Institute, Bijenička 54, HR-10000 Zagreb, Croatia \\ ${ }^{2}$ Institute of Physics, Bijenička 46, HR-10000 Zagreb, Croatia \\ ${ }^{3}$ Elettra-Sincrotrone Trieste, SS 14, km 163.5, I-34149 Basovizza, Italy
}

Correspondence should be addressed to B. Pivac; pivac@irb.hr

Received 15 July 2013; Accepted 13 August 2013

Academic Editors: C. Angeles-Chavez and R. Birjega

Copyright (C) 2013 P. Dubček et al. This is an open access article distributed under the Creative Commons Attribution License, which permits unrestricted use, distribution, and reproduction in any medium, provided the original work is properly cited.

\begin{abstract}
This work analyzes the effect of nanosecond laser pulse deposition of GaAs in an inert atmosphere of Ar and He. The number of pulses and the gas pressure were varied and the effect on the nanoparticles formation was studied by scanning electron microscopy, grazing incidence small angle X-ray scattering, and atomic force microscopy. It is shown that the GaAs nanoparticle sizes and size distributions can be controlled partly by the number of laser pulses applied during their production and partly by the choice of inert gas and its pressure. Our results suggest that $\mathrm{He}$ is a more promising working gas producing narrower size distributions and a better size control of the grown nanoparticles.
\end{abstract}

\section{Introduction}

Pulsed laser deposition (PLD) is a simple and convenient method of producing various types of materials among which are also nanoscaled materials [1]. In such materials the quantum confinement becomes a dominant effect and it significantly modifies their properties. PLD using very short pulses is particularly interesting for the deposition of complex multielement films, preserving the stoichiometry of the parent materials [2]. Numerous experiments were carried out and rich scientific information of the ablation process was obtained. Nevertheless, several mechanisms involved in these processes are not yet completely understood. Different results cause continuing discussion about ultrafast melting [3], resolidification dynamics [4], surface structure modification [5], thermal and nonthermal mechanisms of ablation [6], and direct cluster emission. This is the reason for the continuation of extensive studies, both theoretical and experimental, on the dynamics of laser heating, melting, resolidification, and ablation of the target material irradiated by different pulse durations and wavelengths.
The effects of the background gas on the deposition process were considered in a number of papers [7]. The experimental results on ablation in the presence of ambient gas revealed the importance of the ambience gas parameters, though some of them are not of chemical nature. Previous results [8] show that the effect of the background gas depends on the combination of the ambient and laser parameters used in the PLD. In the PLD process, the nature of the phenomena taking place during the plasma expansion depends upon the gas pressure in the ablation chamber [9]. Under low pressure, from vacuum up to $0.5 \mathrm{mbar}$, atoms and ions are solely present in the plume leading to film formation and growth. At intermediate gas pressure, 1-20 mbar, the emitted species undergo collisions with gas molecules and condensation occurs in the plume leading to the nanosized particles formation. At increasing pressures higher than $100 \mathrm{mbar}$, the nanoparticles tend to aggregate and large "cauliflower" or "snowflake" structures are observed.

As the most important representative of III-V semiconductors, gallium arsenide (GaAs) is quite suitable for use in optoelectronic industry and in particular for high-efficiency 


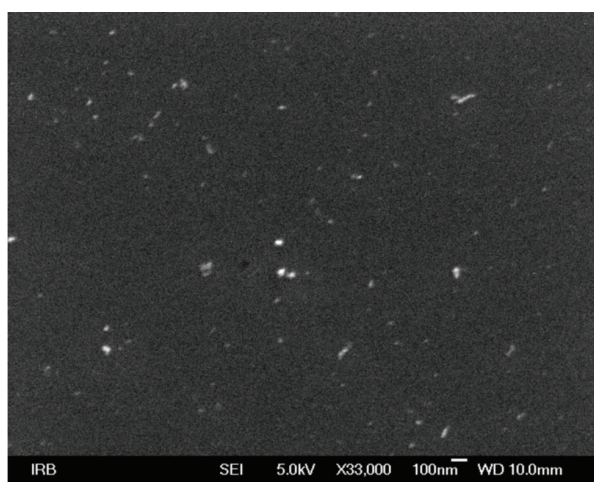

(a)

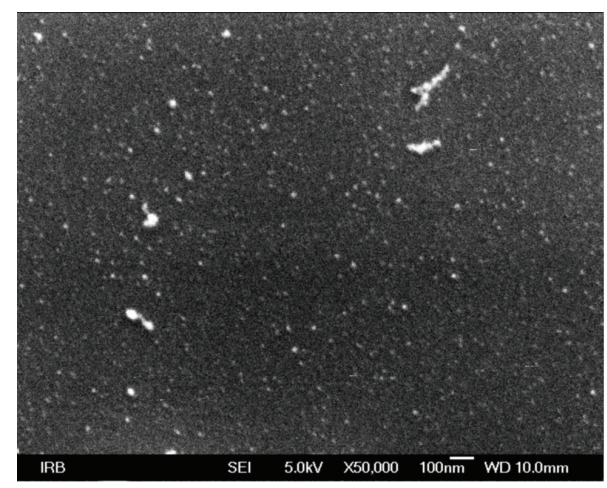

(b)

FIGURE 1: SEM image of 100 (a) and 1000 (b) pulses deposited GaAs at 1 mbar Ar working gas.

solar cell production because of its nearly ideal band gap $(1.43 \mathrm{eV})$ for single-junction solar cells [10]. Having a high optical absorption, it requires a cell only few microns-thick to absorb the sunlight. Another reason such as resistance to heat and radiation damage caused, the GaAs material is preferred for use in the space applications. Furthermore, GaAs nanoparticles with dimensions on the order of only few nanometers have an even greater potential if quantum confinement effects could be used to tune its properties.

The primary scope of this work is to explore the effects of the PLD deposition parameters and/or different working gases used in the process of deposition on the morphology of GaAs nanoparticles deposited on Si substrate. The idea was to deposit and explore isolated nanoparticles instead of continuous films.

\section{Experimental}

The GaAs nanostructures were deposited on clean Si substrates in a PLD setup with the fundamental emission at $1064 \mathrm{~nm}$ of a Q-switched neodymium doped yttrium aluminum garnet laser with 4 ns pulse length and $5 \mathrm{~Hz}$ repetition rate. The substrate was p-type $\mathrm{Si}(100)$ wafer and the target was intrinsic GaAs, crystal orientation (100). The details of the deposition process are given in [8]. The deposition was carried out under flow of argon or helium (both of 99.99\% purity) at a given pressure with a base pressure in the vacuum chamber of $8 \times 10^{-5} \mathrm{mbar}$. The substrate was not heated during deposition in order to avoid interface stress and deformation during cooling. The target was exposed to a laser pulse energy of $140 \mathrm{~mJ}$ (pulse fluence was $31 \mathrm{~J} / \mathrm{cm}^{2}$ ), resulting in GaAs particles formation on the Si substrate.

The GISAXS experiments were carried out at the synchrotron facility Elettra (Trieste, Italy) at the SAXS beamline, using radiation with wavelength $\lambda=0.154 \mathrm{~nm}$ (i.e., with a photon energy of $8 \mathrm{keV}$ ). A thin Al-strip was placed in front of the $2 \mathrm{D}$ detector to avoid its saturation in the specular plane direction where the usually much stronger surface scattering is present. The spectra were corrected for background intensity and detector response. Details about the GISAXS geometry and data analysis are given elsewhere [8].
AFM measurements were performed with a Nanoscope IIIa controller (Veeco Instruments, Santa Barbara, CA) with a vertical engagement (JV) $125 \mu \mathrm{m}$ scanner, using a silicon tip (TESP, Veeco) that had $8 \mathrm{~nm}$ nominal radius.

The scanning electron microscopy (SEM) images were produced using the JEOL JSM-7000F field mission SEM.

\section{Results and Discussion}

Figure 1 showing SEM images of two samples, deposited with 100 (a) and 1000 (b) pulses in Ar under 1 mbar pressure, illustrates the effect of PLD deposition in two extreme cases. Well-defined, separate particles of diameters around 10$20 \mathrm{~nm}$ are clearly resolved in 100 pulses sample, while the number of particles is much greater after 1000 pulses, and the substrate appears to be covered completely. In addition, few bigger, agglomeration-like structures are present on both samples. As particle heights are not recoverable from this type of measurements, GISAXS and AFM are applied for full particle analysis.

The results of GISAXS measurements on PLD deposited GaAs nanoparticles on monocrystalline silicon substrate, produced by 100,500 , and 1000 laser pulses, are shown in Figure 2. The figure presents the results from $\mathrm{He}$ assisted deposition at the working gas pressure of $1 \mathrm{mbar}$. Due to the isotropy of the film structure, the measured scattering intensity was symmetrical with respect to the specular plane $\left(q_{y}=0\right)$. Making use of this symmetry we represent in the left side of images the measured intensity, while the right side shows the result of data simulation. The very good symmetry of the two parts confirms the simulation quality and the selection of parameters, as it will be discussed below.

The grazing incident angle was set to the critical angle value for $\mathrm{GaAs}$, therefore enhancing the scattering from the deposited thin film, since the X-ray beam within the sample was concentrated close to the surface and the penetration depth was less than $20 \mathrm{~nm}$. The reduced intensity at the central part of the measured intensity (at $q_{y}=0 \mathrm{~nm}^{-1}$ ) is due to a semitransparent absorber that was placed there in order to reduce very high intensities appearing in the specular plane. Using the absorber, the dynamic ratio of the measured intensities could be enhanced, and the detector was protected 


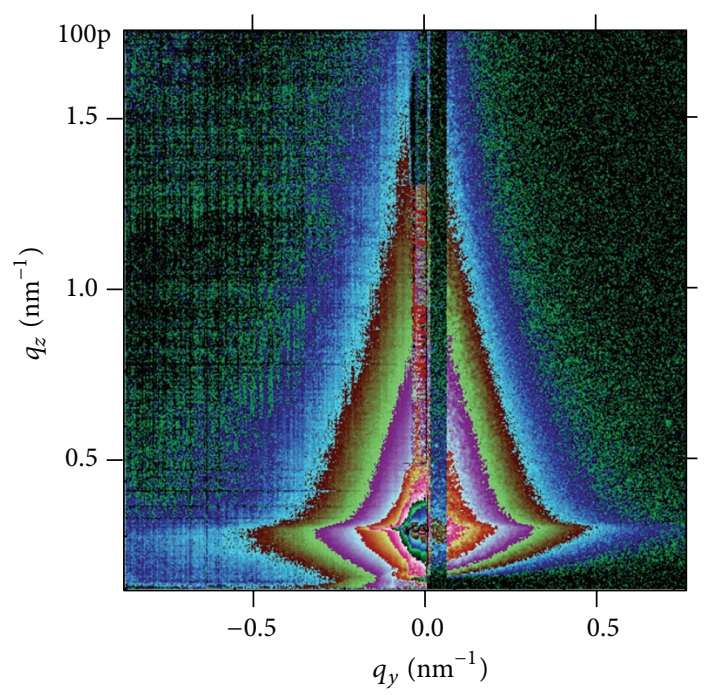

(a)

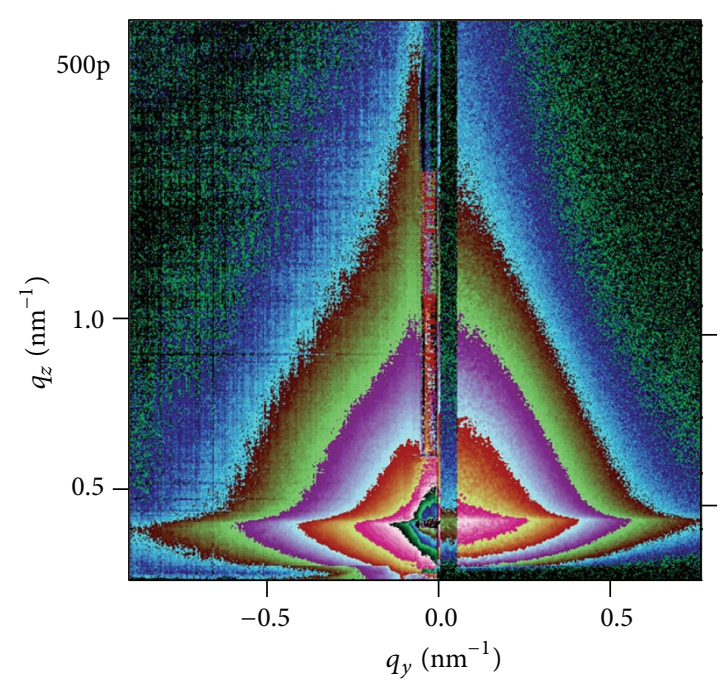

(b)

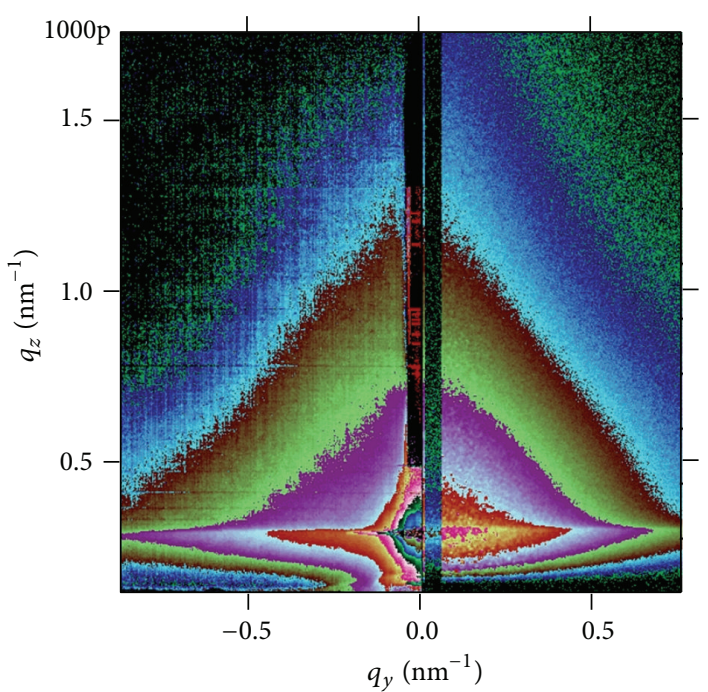

(c)

FIGURE 2: GISAXS intensity pattern for He (1 mbar) assisted PLD of GaAs. The number of pulses is increasing from top to bottom, that is, (a) 100, (b) 500, and (c) 1000. The left side of each image represents the measured intensity, while the right side is the result of numerical fitting. 


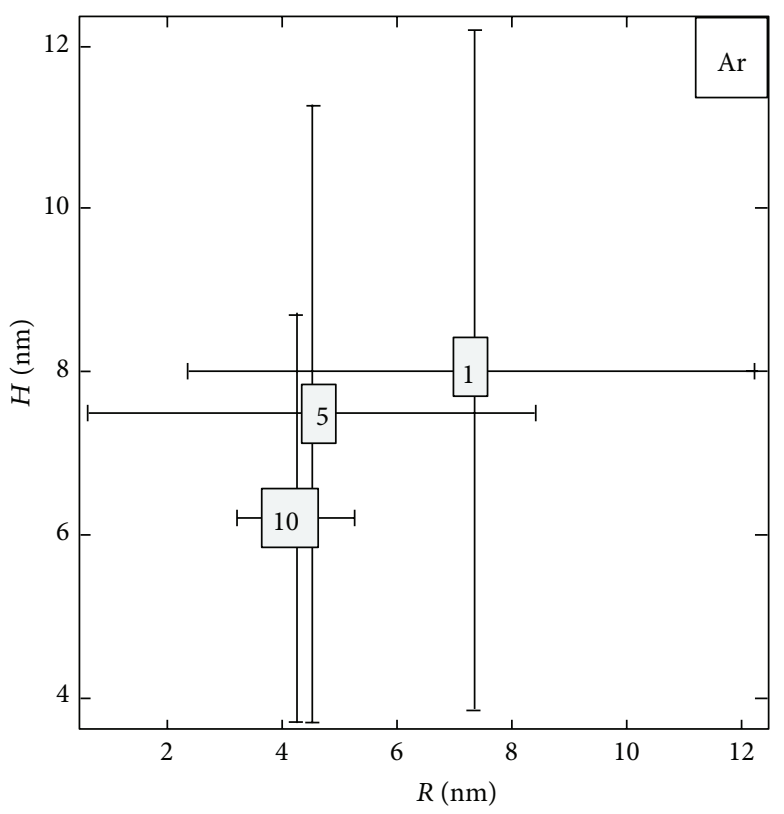

(a)

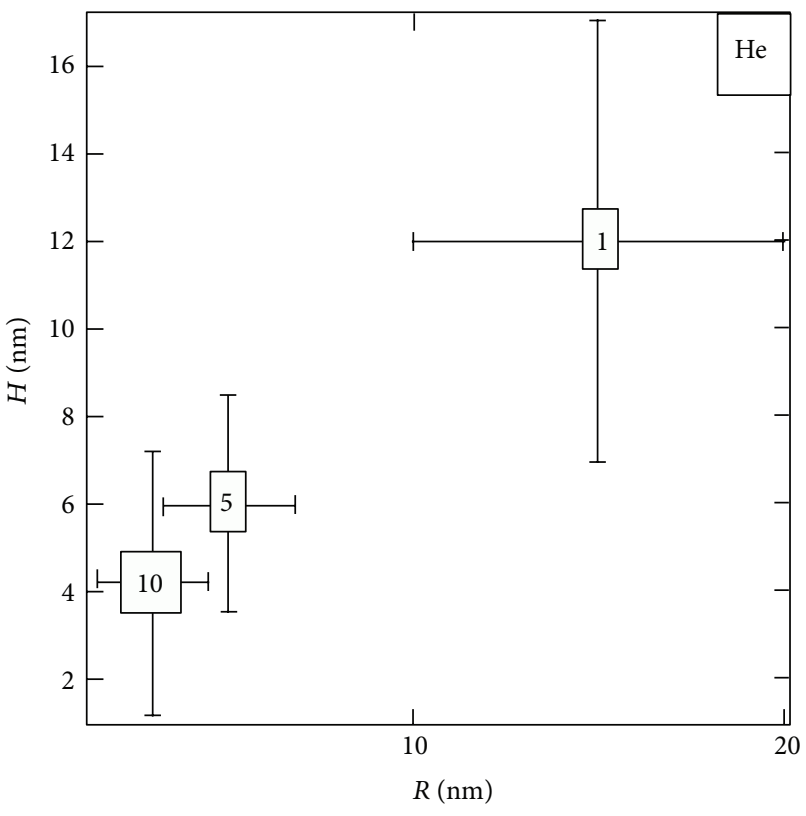

(b)

FIGURE 3: Results of log-normal size distribution fits to the experimental GISAXS data, for Ar and He assisted deposition left and right, respectively. The height $H$ versus radius $R$ is plotted. The size ranges resembling error bars are actually size distribution widths. The numbers on the graphs indicate the pulse number (to be multiplied by 100).

from saturation. The observed intensity maximum, spread horizontally at about $q_{z}=0.25 \mathrm{~nm}^{-1}$, common to all samples, is due to the Yoneda peak [11]. Since the grazing angle was set to the critical angle value, the maximum (peak) intensity in $z$ direction is at this position. This is also where the specular peak is situated (at $q_{y}=0$ where the grazing angle equals half of the scattering angle). Finally, the scattering from the largest objects on the sample (i.e., on the surface) is concentrated here. The intensity area above the Yoneda peak, where the information about the film topology dominates, is further used in the analysis.

Figure 2 clearly shows the changes in shape of the measured intensity distribution (left part of each image) from the top to the bottom as the number of pulses is increased. It is interesting to note that the apparent difference between 100 and 500 pulses (fivefold increment) is less pronounced than from 500 to 1000 (twofold increment). Moreover, similar patterns at the central part (at about $q_{y}=0 \mathrm{~nm}^{-1}$ ) of all images suggest that the particles are not closely packed and/or the positions of the particles are uncorrelated.

By calculating the scattering from a distribution of particles, located on the surface of a substrate, applying our own software, the numerical data fitting to the measured data was obtained. We applied a model of either cylinders or domes, with diameter and height as the parameters according to the log-normal size distribution, in the numerical reproduction of the measured intensities. The results of the simulations are displayed at right part of each image. The very good symmetry between left and right sides indicates the quality of the fit.
It is shown that the shape of the modeled scattering patterns could reproduce the measured ones properly only when appropriate particle shapes were applied. The simulation results, shown in Figure 2, were obtained applying a cylindrical shape for 100 and 500 pulses and domes for 1000 pulses.

The numerical results of the simulations are summed in Figure 3, where the results obtained by PLD in He atmosphere discussed here are compared to those obtained previously in $\operatorname{Ar}$ [8]. The average values obtained for particle radii and heights are plotted for the different pulse numbers, and the error bars are representing the corresponding log-normal distribution half widths. Both horizontal radii $(R)$ and vertical heights $(H)$ are shown for Ar (left) and He (right) working gas. There is a clear trend of size reduction as the number of pulses is increased, which is less pronounced for Ar than for He. The horizontal radius falls from $15 \mathrm{~nm}$ to $3 \mathrm{~nm}$ and the height from $12 \mathrm{~nm}$ to $4 \mathrm{~nm}$ in the He atmosphere as opposed to the reduction from $7 \mathrm{~nm}$ to $4 \mathrm{~nm}$ in radius and from $8 \mathrm{~nm}$ to $6 \mathrm{~nm}$ in height, in the case of Ar. Another remarkable difference between the two working gasses lies in the relative half width of the size distribution. Ar working gas results in broader size distributions: for example, the average horizontal radius for 500 pulses is $4.5 \mathrm{~nm} \pm 4 \mathrm{~nm}$, as opposed to $5 \mathrm{~nm} \pm$ $2 \mathrm{~nm}$ in the He case.

We conclude that for 1000 pulses a homogeneous film has been formed, and the modeled size distribution is describing the surface topology/roughness of this film. Although the shape of the scattering pattern appears to be similar for 100 and 500 pulses (compare [8]), the extreme value of the size distribution width obtained for 500 pulses in the case of 


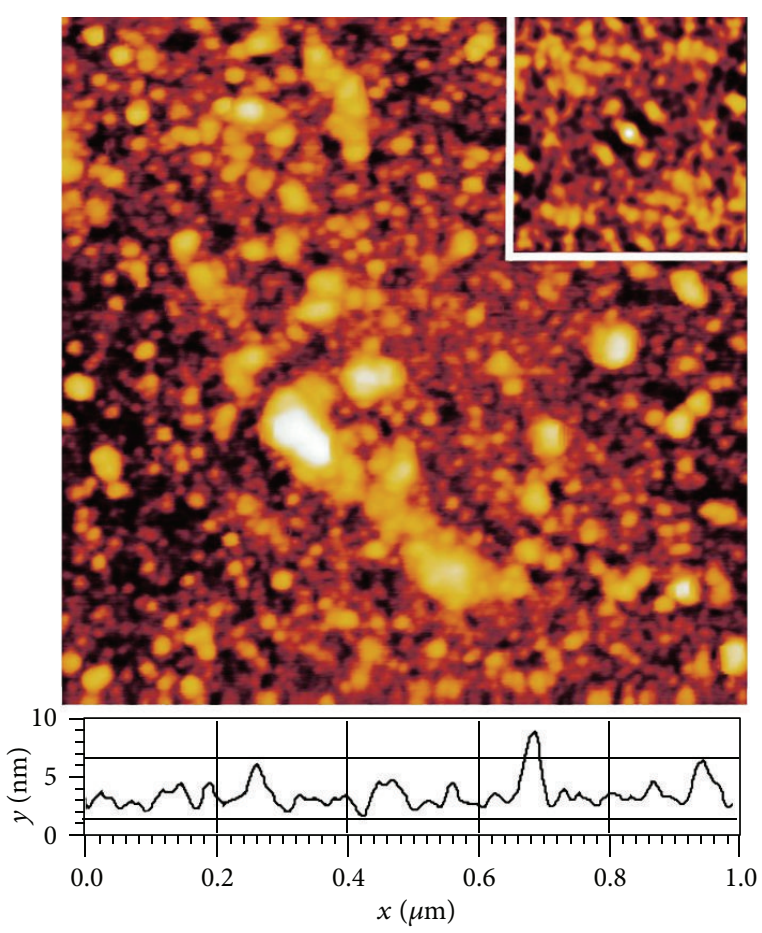

- He 100p1

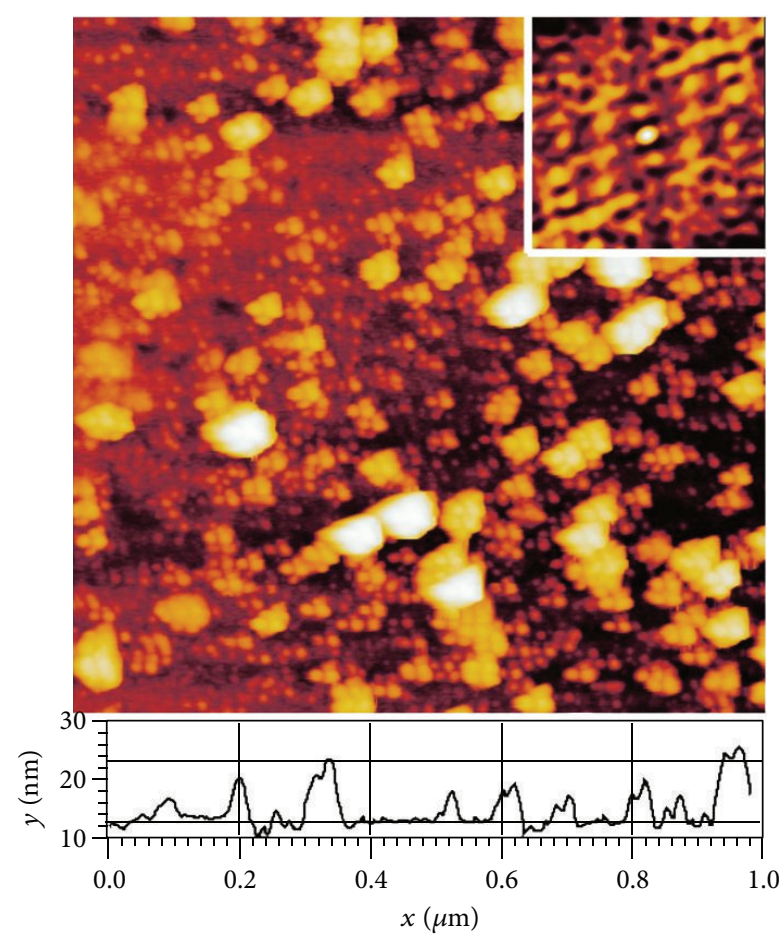

— He 500pl

(a)

(b)

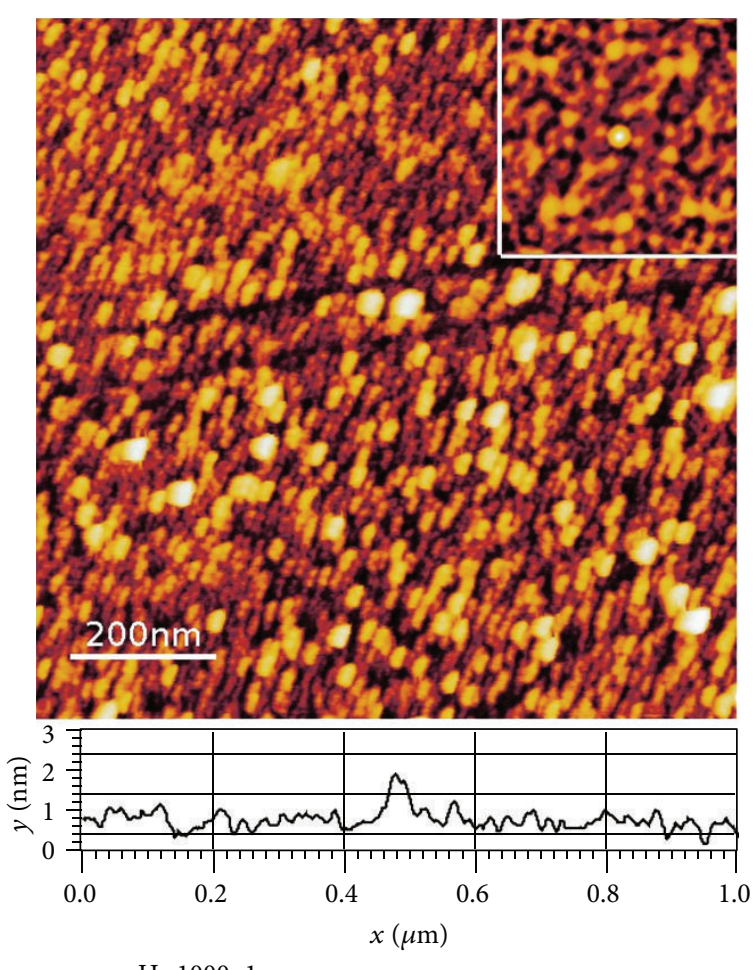

- He 1000p1

(c)

Figure 4: AFM images of the He assisted PLD of GaAs. Number of pulses: (a) 100, (b) 500, and (c) 1000 . The image sizes are $500 \times 500 \mathrm{~nm}{ }^{2}$, and the insets show the corresponding autocorrelation functions. Below each figure is typical line scan of $2 \mathrm{D}$ image. 


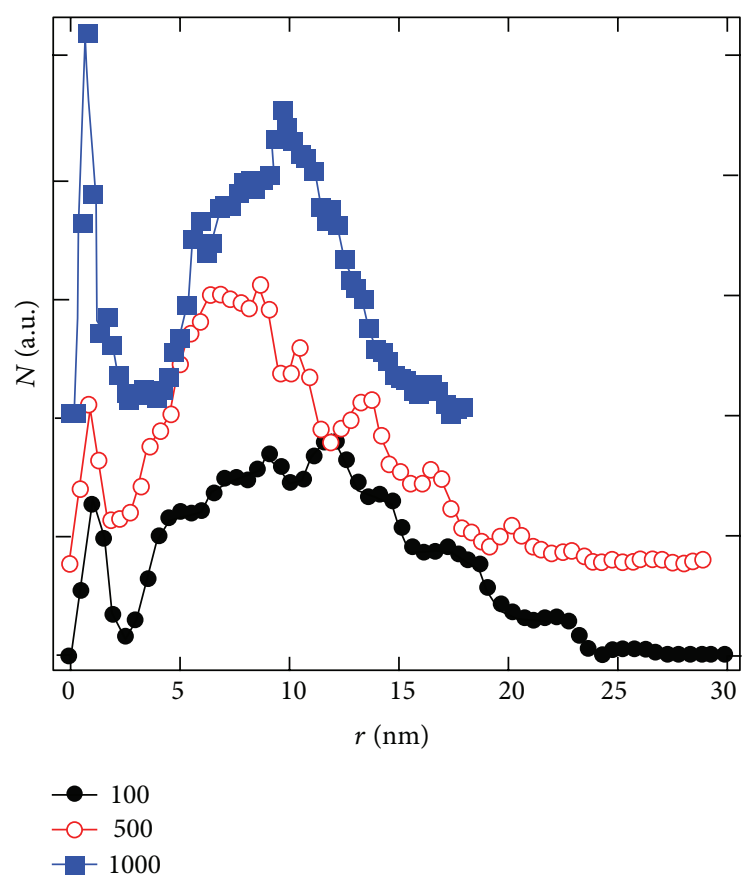

(a)

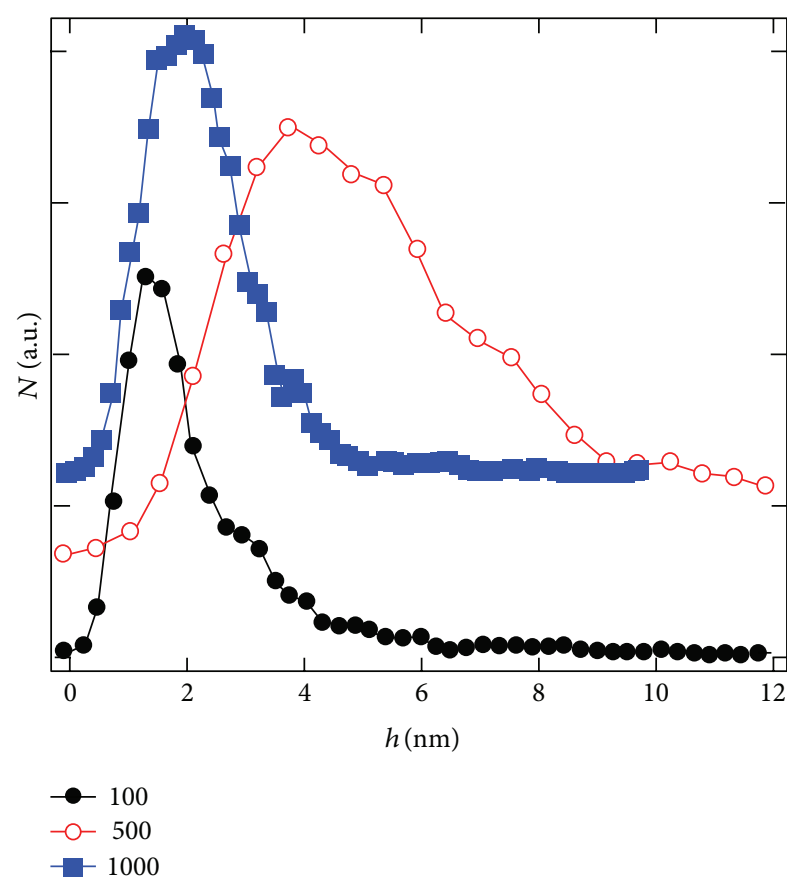

(b)

FIGURE 5: Size distributions obtained from AFM data by the water shed algorithm, as explained in the text: equivalent horizontal radius (a) and particle height (b) with number of pulses as the parameter indicated in insert.

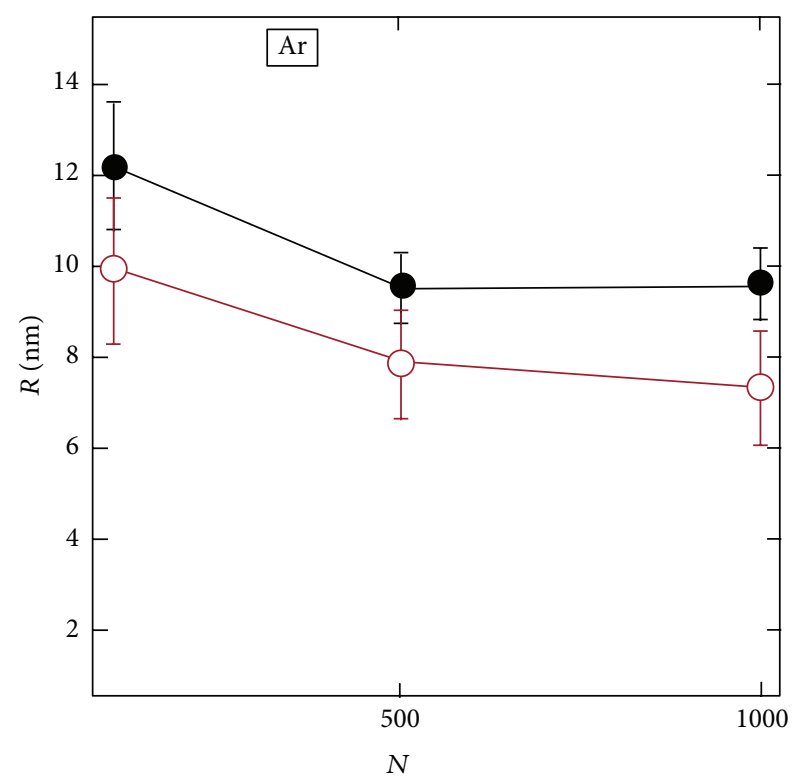

(a)

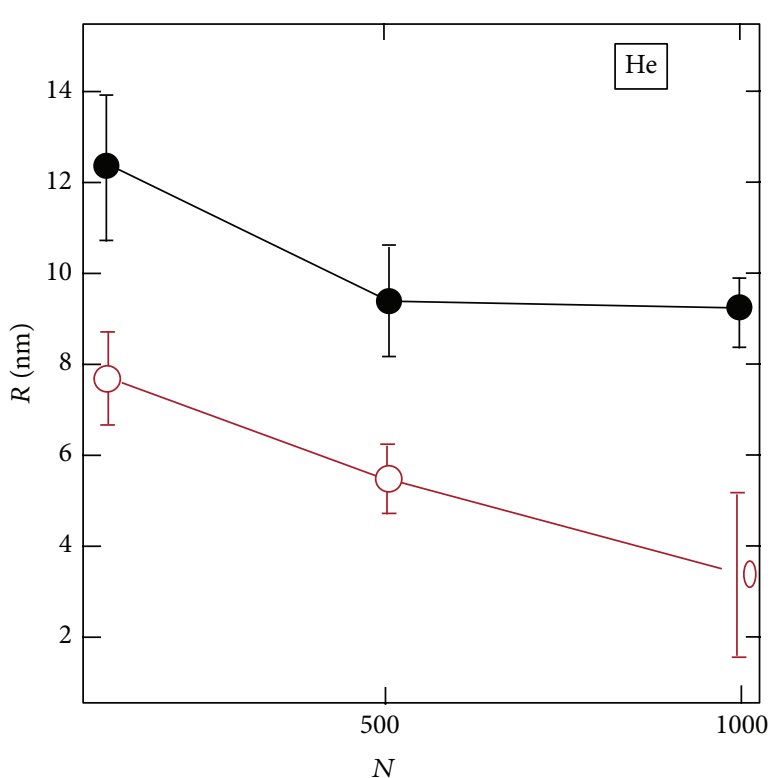

(b)

FIGURE 6: Average sizes (full circles) and size distribution widths (open circles) versus number of pulses, obtained from AFM data by fitting to a log-normal distribution. For comparison purposes, the values obtained for Ar are also shown. 

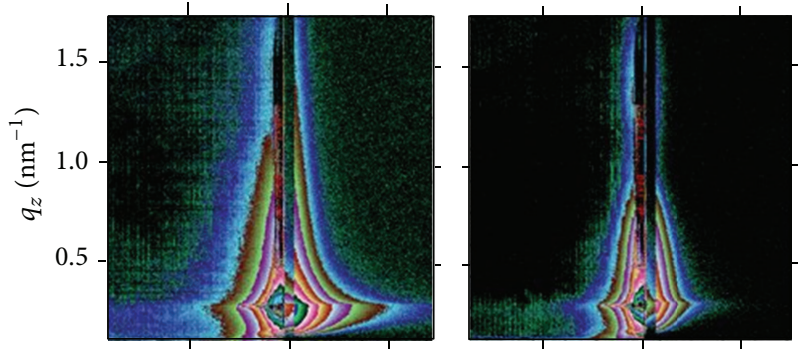

(a)
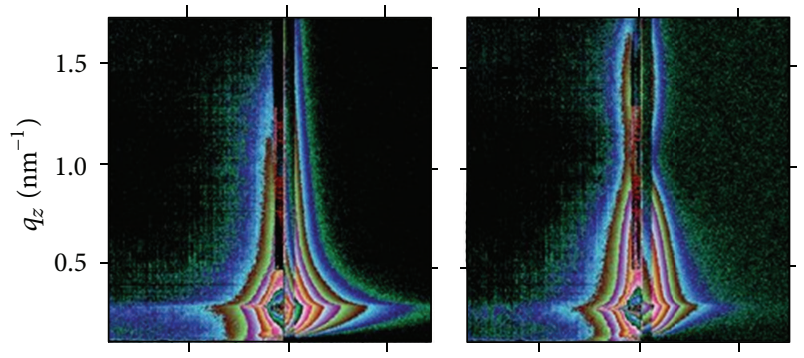

(b)
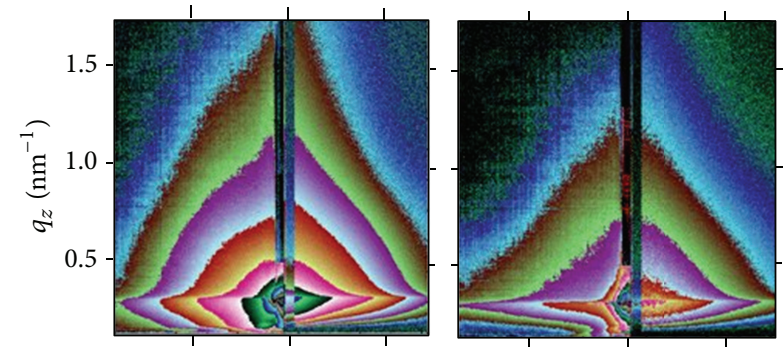

(c)
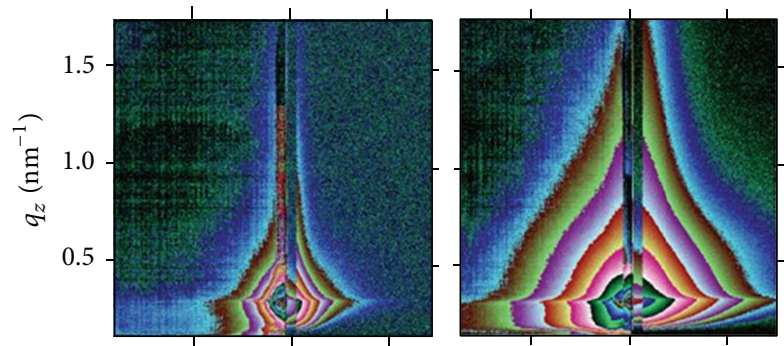

(d)

FIGURE 7: GISAXS intensity pattern for Ar and He assisted PLD (1000 pulses) of GaAs left and right, respectively. The working gas pressure is increasing from top to bottom, that is, (a) $0.01 \mathrm{mbar}$, (b) $0.1 \mathrm{mbar}$, (c) $1 \mathrm{mbar}$, and (d) $10 \mathrm{mbar}$. The left side of each of the images represents the measured intensity, while the right side is the result of a numerical fit.

Ar background gas indicates that the change of the particle shapes begins already here. The scattering from the 100 and 500 pulses samples contains a rather strong contribution from the substrate surface, but this is mostly restricted to the specular plane and partly hidden below the abovementioned absorber and thus has been ignored in analysis.

The in-plane particle-to-particle correlation was not included in the fitting function since no depletion of the intensity in the central part has been observed. Thus correlation can only play a minor role when the size distribution is very wide.

To obtain a better insight into the nanoparticles/film formation, the samples have been also characterized by AFM. Figure 4 shows AFM images of the same samples from Figure 2. A gradual increment of the number of particles as the number of pulses is increased from 100 to 1000 can be seen: in the first two samples (100 and 500 pulses) the particles are dispersed, while in the last they are packed more densely.

Agglomeration of the particles can be also seen after 500 and 1000 pulses in Ar [8]. Still it is not completely clear whether this is a result of material transport directly from the target instead of deposition from plasma or it can be ascribed to some irregularities in the deposition from the plasma itself, but this has been noted also before [12]. Nevertheless, the size of these agglomerates is typically above a few $\mu \mathrm{m}$ and therefore they could not be detected in our selected GISAXS range.

The watershed algorithm was used to locate and count the particles on the surface (i.e., numerically simulating water redistribution over the surface due to surface roughness). The distributions of particle equivalent disk radius and height are determined and plotted in Figure 5. The equivalent radius of a particle is calculated from the disk area that equals the area of the particle projection on the substrate, while the height is the maximum height value in the particle, regardless of the actual shape. A log-normal size distribution fit was applied to the results and the fit parameters are displayed in Figure 6. The general trend follows the one from the GISAXS results: both the size and distribution widths are decreasing with the number of pulses. The average particle size is bigger by about $4 \mathrm{~nm}$ with respect to those obtained from GISAXS; only the value for the 100 pulses sample in He is slightly lower. However, AFM suggests a substantially wider size distribution for all samples. Generally, the size distribution width is narrower in samples with He working gas, both in GISAXS and AFM results. However, the particle heights obtained by AFM have much smaller values than those obtained by GISAXS: 1.5 and $2 \mathrm{~nm}$ for 100 and 1000 pulses, respectively, and only 500 pulses particles have the height comparable to their radius (see Figure 5).

Autocorrelation functions, calculated from the AFM data for each image, are shown as inserts in the upper right corner of the AFM images in Figure 4. We can see that the autocorrelation symmetry is of the second or fourth order, which suggests a rather strong influence of the substrate which is (100) oriented monocrystalline Si. However, the lattice missmatch between $\mathrm{Si}$ and GaAs is substantial, and self-organization was very limited. The samples deposited at 100 and 1000 pulses in Ar atmosphere have an autocorrelation function that displays side maxima in only one direction [8], while the autocorrelation function of the 500 pulses $\mathrm{He}$ sample has a symmetry close to the sixth order suggesting some self-organization that results from an isotropic surface force which is partly present.

We have further investigated the influence of the working gas pressure on deposition and formation of GaAs nanodots. For this purpose, two series of samples, using $\mathrm{Ar}$ and $\mathrm{He}$ 


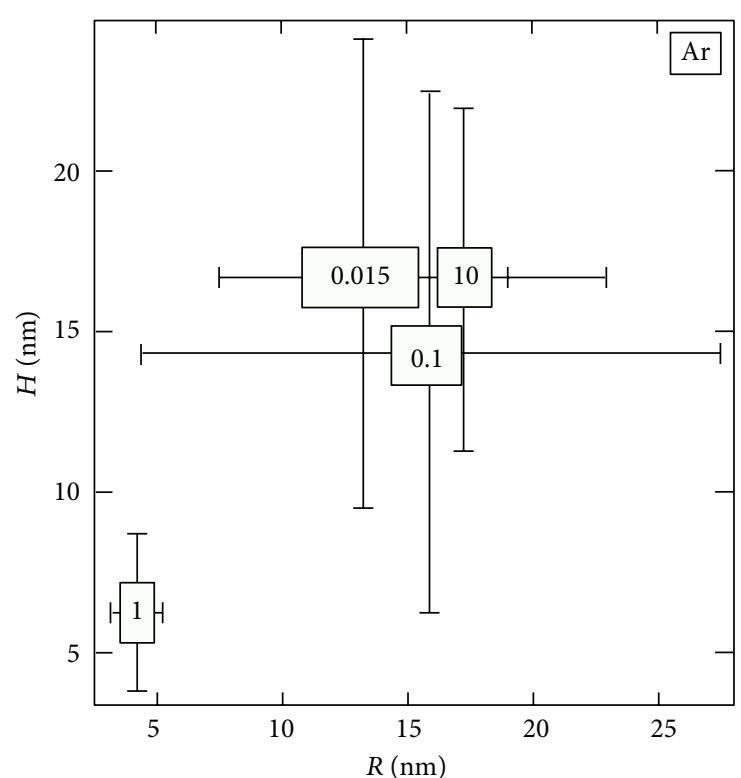

(a)

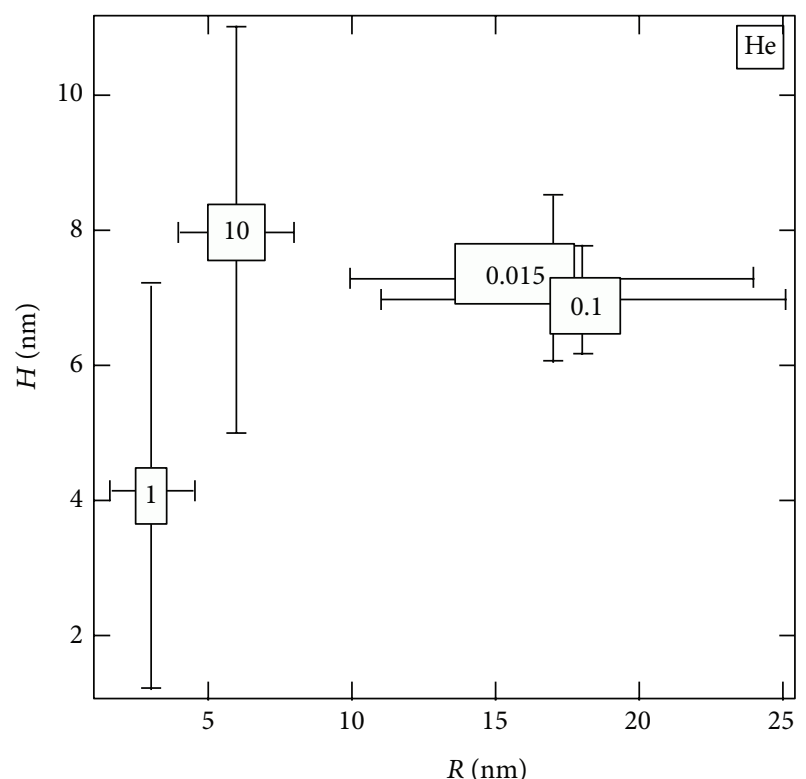

(b)

FIGURE 8: Results of log-normal size distribution fits to the experimental data versus working gas pressure, for Ar and He assisted deposition left and right, respectively. The height versus radius is plotted. The size ranges resembling error bars are actually size distribution widths. The numbers on the graphs indicate the gas pressure in mbar.

atmosphere, have been prepared under pressures of $0.015,0.1$, 1.0 , and 10 mbars, while the number of pulses was always 1000 . For these, the GISAXS intensity distributions are shown in Figure 7. The left and right column correspond to deposition in $\mathrm{Ar}$ and $\mathrm{He}$ atmosphere, respectively. The pressure is increasing from top to bottom. Again, only the left side of each of the GISAXS images represents the measured data, while the right side is the result of the best fit to the measured values. Note that the third row for He working gas $(1 \mathrm{mbar}$ data) is a repetition of the last row of Figure 2.

For the two lower pressures, the GISAXS data almost resemble those obtained for the lowest number of pulses, suggesting that the film thickness/nature is similar. Indeed, we have been able to fit the data successively using a cylindrical particle shape in our model. The $1 \mathrm{mbar}$ row has already been discussed in connection with Figure 2. Here we had to assume a hemispherical shape to obtain good fit, and this also holds for the highest pressure data.

As expected, the difference between the two working gasses is minimal for the lowest pressure $(0.015 \mathrm{mbar})$ and it diminishes completely with a further pressure reduction. On the other side, the GISAXS signal is more spread over the detector angular range, indicating smaller particle sizes. However, this does not hold for the 10 mbar Ar deposited sample, where the particle sizes are again larger. The overall trend for He deposited samples seems to be a smoother function of the pressure. In addition, at the two lower pressure values, the GISAXS signal exhibits oscillations in vertical direction, a clear indication of particle height uniformity over the sample surface. Also, in-plane particle-to-particle correlation was not included in the fitting function since no depletion of the central intensity part has been observed.
Apparently, at low working gas pressure, a rather homogeneous, well-defined film was formed. Actually, what we have seen by GISAXS are details of the inner film structure and columnar growth. This is approximated by cylinders rather easily during the data fitting. The particle height is a good measure of the film thickness. As the gas pressure is increased, the growth of particles of diverse size is favored, since the energy of the atoms deposited from the plasma is reduced. In this way, atoms are sticking to the surface very close to the place of arrival, and, since the rearrangement is lower, a less compact film is formed.

The results of log-normal size distribution fits versus working gas pressure are shown in Figure 8 as plot of $H$ versus $R$. As well as in Figure 3, the error bars are actually the corresponding log-normal distribution half widths, and the left and right graphs display the influence of the $\mathrm{Ar}$ and $\mathrm{He}$ working gas, respectively.

The horizontal radius has similar values and trends for both working gasses: there is a small increment of the radius going from 0.015 to $0.1 \mathrm{mbar}$, and the values are minimal for $1 \mathrm{mbar}$, then the radius increases again for $10 \mathrm{mbar}$, although this increment is significant only in the case of Ar. The half width of the horizontal radius distribution is also minimal for $1 \mathrm{mbar}$ pressure for both working gasses, while the maximum is reached for $0.1 \mathrm{mbar}$.

However, the most striking difference between $\mathrm{Ar}$ and $\mathrm{He}$ assisted deposition can be seen in the particle height values: in He working gas they are half the values obtained for Ar. Otherwise, the trend is similar: the values are decreasing with pressure up to $1 \mathrm{mbar}$ and then they rise again to $10 \mathrm{mbar}$, which actually is the maximum value. As in the case of horizontal radii, the minimum is reached for $1 \mathrm{mbar}$ 

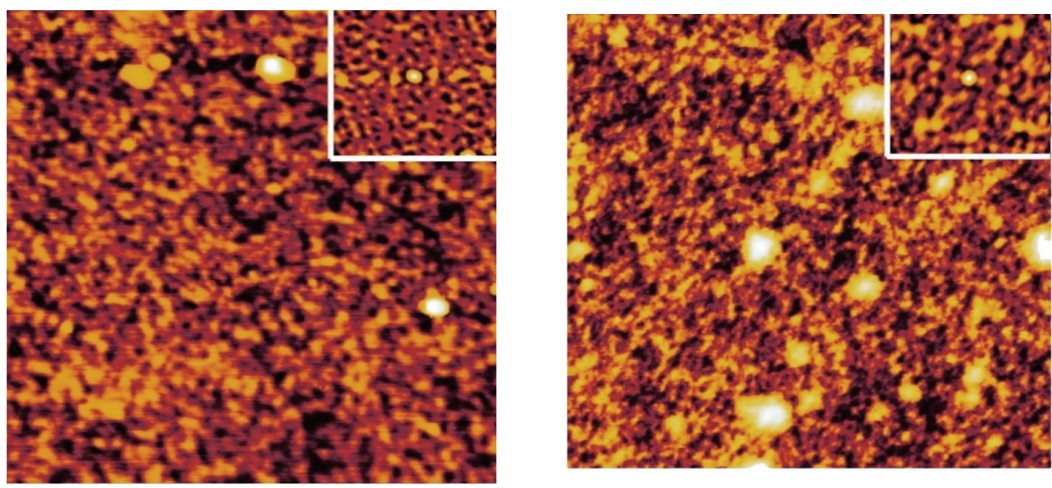

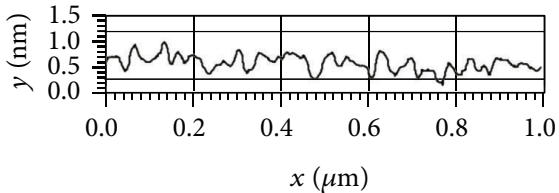

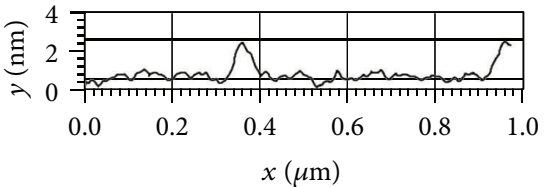

— Ar 1000p001

— He 1000p001

(a)
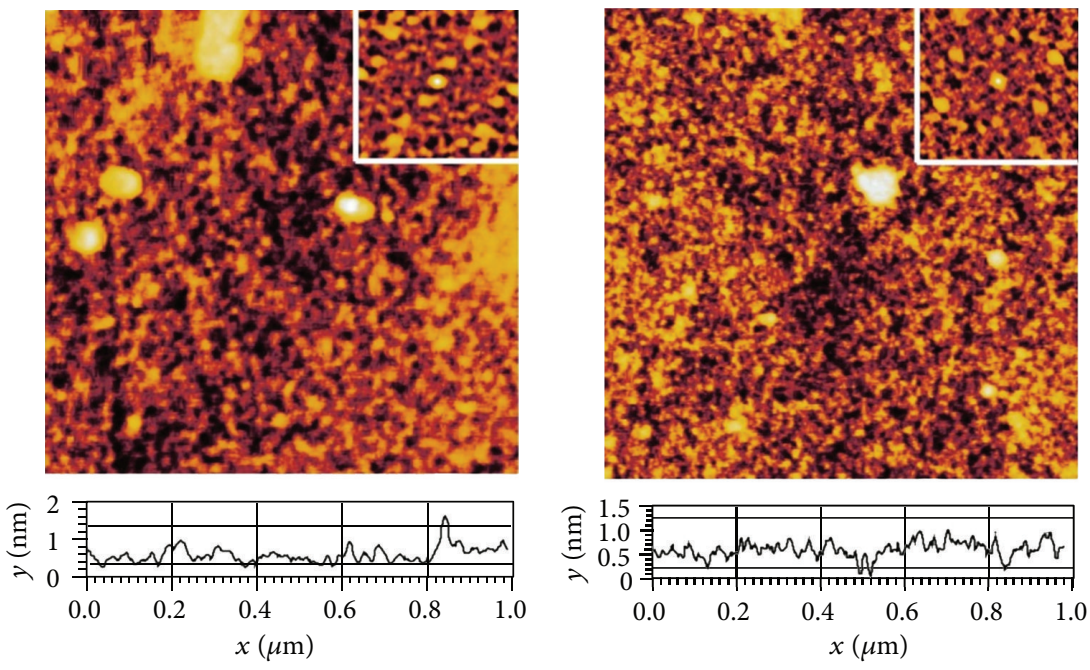

- Ar 1000p01

— He 1000p01

(b)
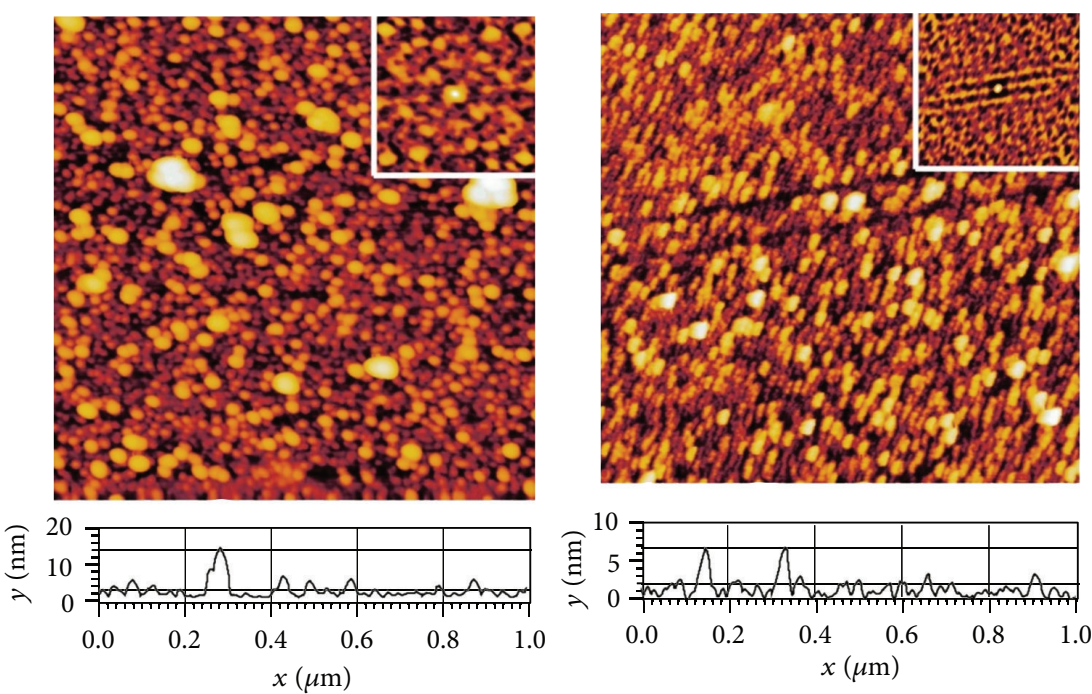

- Ar 1000p1

— He 1000p1

(c)

FIgure 9: Continued. 


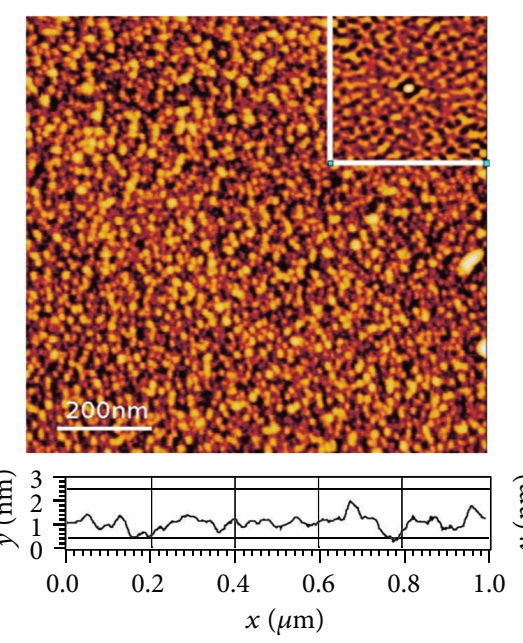

- Ar 1000p10
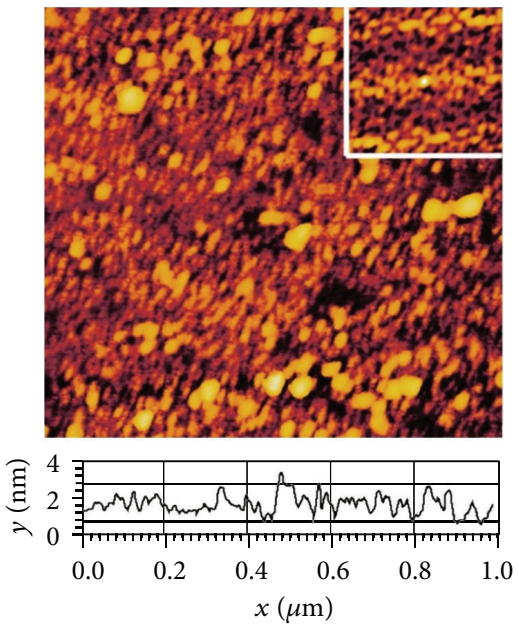

- He 1000p10

(d)

Figure 9: AFM images of the Ar and He assisted PLD of GaAs left and right, respectively. The working gas pressure is increasing from top to bottom, that is, (a) $0.01 \mathrm{mbar}$, (b) $0.1 \mathrm{mbar}$, (c) $1 \mathrm{mbar}$, and (d) $10 \mathrm{mbar}$. The insets show the corresponding autocorrelation functions.

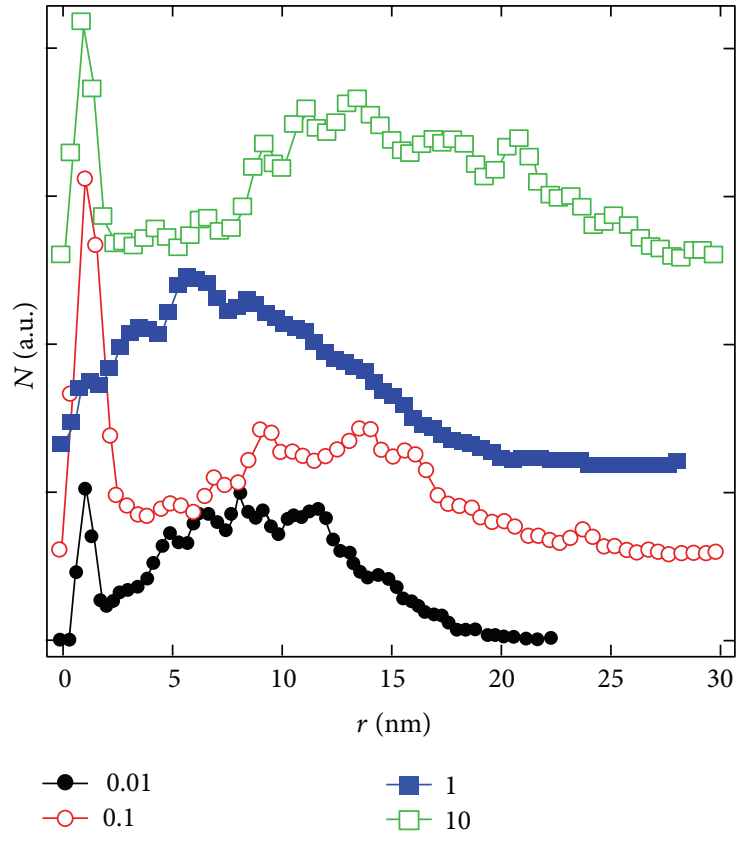

(a)

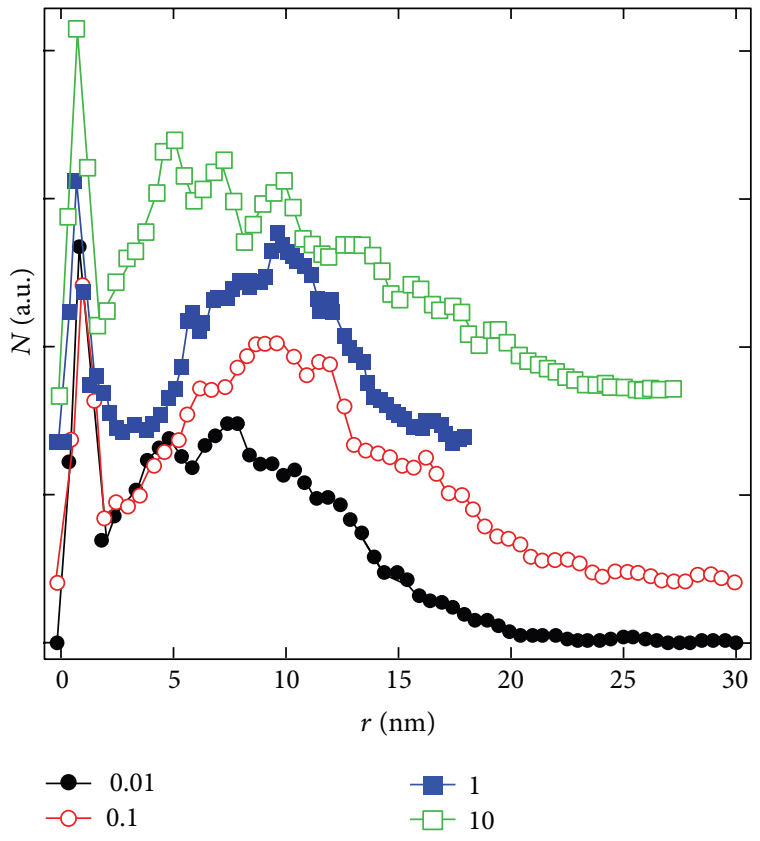

(b)

FIGURE 10: Equivalent horizontal radius distribution obtained from AFM data for $\mathrm{Ar}$ (a) and $\mathrm{He}$ (b) with working gas pressure as the parameter indicated in insert.

pressure. Height size distributions are generally narrower in He working gas (1 mbar value being the only exception), a more significant difference than in sizes: for example, for 0.1 mbar He size distribution is five times narrower than in Ar case.

The results of the AFM investigation of the sample texture as a function of the working gas pressure are shown in Figure 9 where the AFM images of the same samples from Figure 7 are shown arranged in the same manner: the left and right column represent the $\mathrm{Ar}$ and $\mathrm{He}$ working gas, respectively, while the pressure is $0.015,0.1,1$, and 10 mbars, top to bottom. Again, AFM profiles are plotted below the corresponding AFM image, and autocorrelation images are inserted in the upper right corner of each surface image.

For the lowest two pressures the AFM images show similar features, as one would expect since the lower the pressureis, the closer the growth conditions are to those of growth in vacuum: the mean free path of the particles 


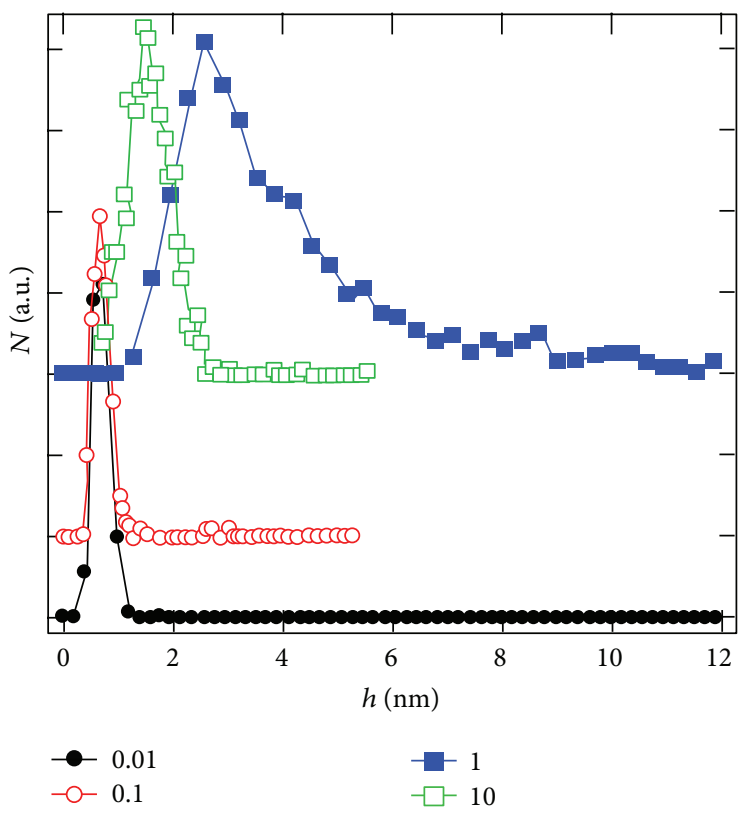

(a)

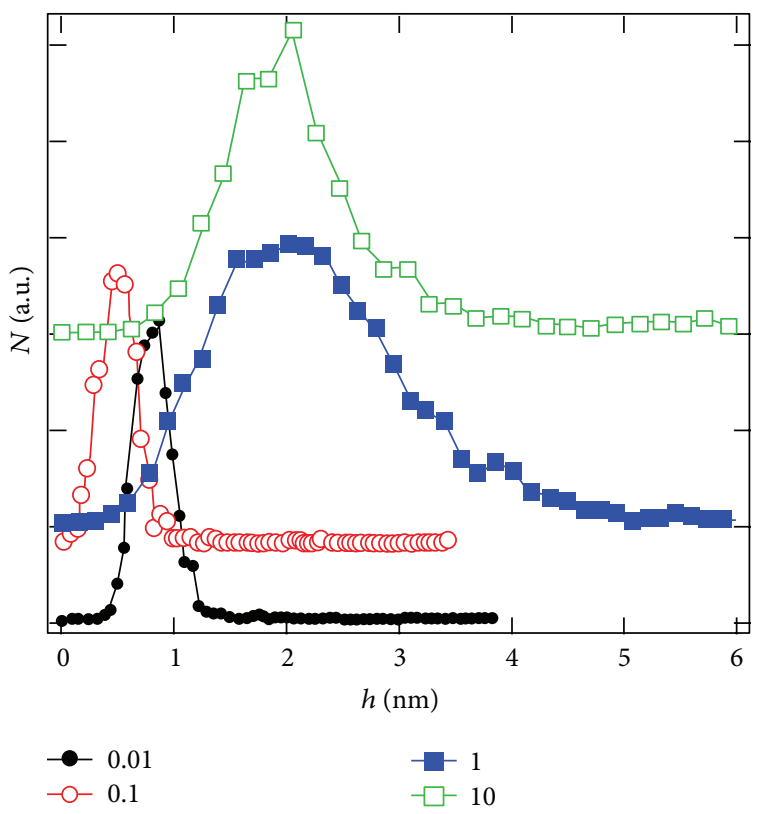

(b)

FIGURE 11: Particle height distribution obtained from AFM data for $\mathrm{Ar}(\mathrm{a})$ and $\mathrm{He}(\mathrm{b})$ with working gas pressure as a parameter indicated in insert.

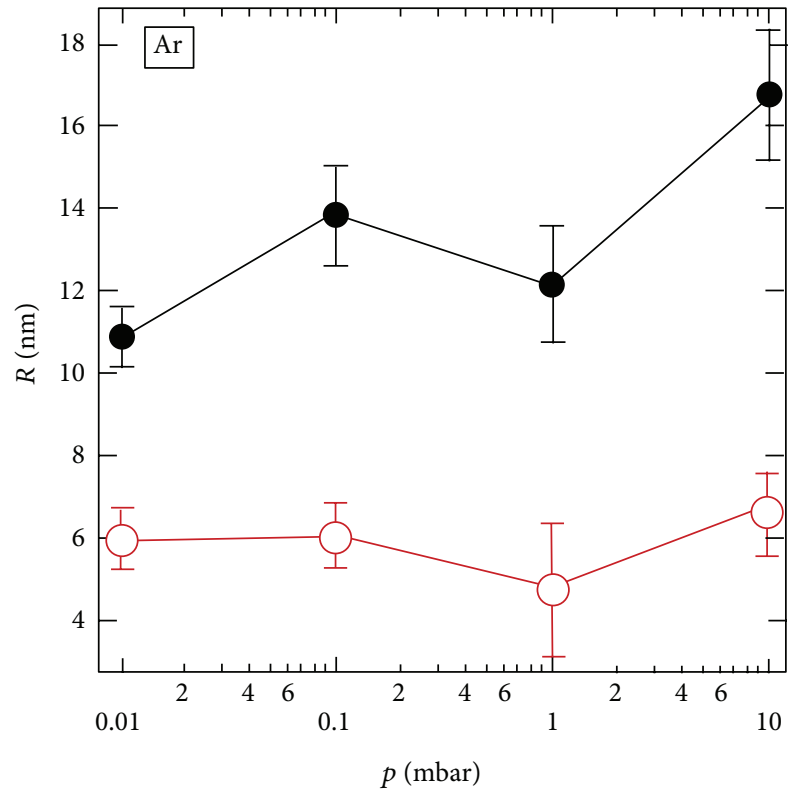

(a)

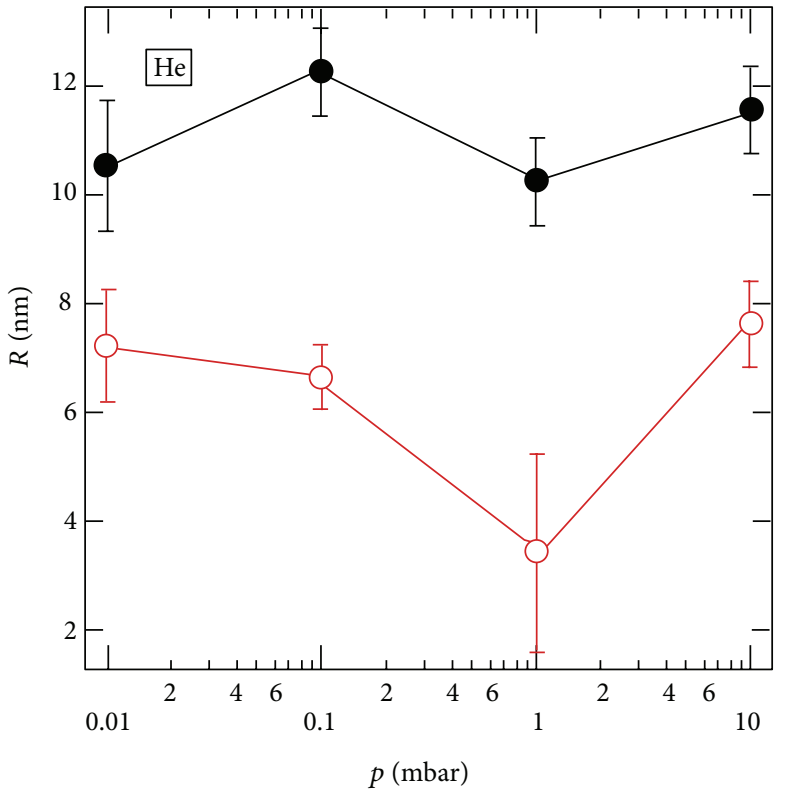

(b)

FIGURE 12: Average sizes (full circles) and size distribution widths (open circles) versus working gas pressure, obtained from AFM data by fitting to log normal distribution.

is longer and the particle energy at impact to the surface is higher, allowing for a better rearrangement and more compact film growth. The particles on the surface appear to be scarcer and the surface roughness is less than a nanometer (compared with the surface profiles for the upper two rows in Figure 9). The good parts of the autocorrelation images show mostly the first order symmetry. As discussed above, the significant lattice mismatch between Si substrate and GaAs film should result in poor particle orientation relative to the substrate, and any ordering should be a result of 
self-assembly. However this is partly evidenced only in the 0.1 mbar He sample, and even here it is rather long ranged and therefore not seen in GISAXS.

As before, the watershed algorithm was used to numerically detect all the particles on the surface, and the results for the particle radii and heights are plotted in Figures 10 and 11 , respectively. Generally, all the particle radii appear to vary in a significantly broad range, but the average value varies more substantially as a function of working gas pressure. However, it is not a simple function of the pressure: it grows with pressure, but exceptions are the $0.1 \mathrm{mbar} \mathrm{Ar}$ and $10 \mathrm{mbar}$ He distribution values.

For comparison, the values of the in-plane particle radii and their corresponding size distribution widths are plotted in Figure 12. We can see that the particle radius follows the pressure in a similar manner for both gasses: it grows with the pressure, but there is a drop in value at 1 mbar. However, the size distribution remains virtually constant, apart from 1 mbar value drop. As mentioned above, overall particle sizes are smaller in He working gas, while the size distribution widths are similar for both working gas cases. One can see that the AFM obtained sizes are similar to those from GISAXS (see Figure 8); only the drop in 1 mbar values is less pronounced.

The low surface roughness for the lower pressure growth has already been mentioned above, and it is evident in Figure 11: particle heights are around $1 \mathrm{~nm}$ for the lower two pressure values in both working gasses. For higher pressure values it increases substantially, but it does not come close to the values obtained by GISAXS.

This discrepancy can, at least partly, be attributed to the difference in probe size, namely, while the X-ray wavelength is $0.154 \mathrm{~nm}$, the AFM tip radius is about $10 \mathrm{~nm}$. Therefore, in some cases where the particle size falls between the two probes sizes, the particle shape is convoluted with the AFM tip shape, while its dimensions are clearly resolved by GISAXS. This discrepancy is the heaviest in the few nanometer range, in which most of the particles in our samples fall. This holds both for particle size and interparticle distance. In case of particles of only a few $\mathrm{nm}$ in size, distributed rather evenly throughout the surface, in a way that the particle-to-particle distance is typically a few $\mathrm{nm}$, the size of the AFM tip hinders a proper height detection more heavily than that of lateral size. When a compact film, with well-defined thickness is formed, AFM is sensitive to sparse irregularities at the surface, and the probing by this method does not penetrate into the film itself. On the other hand, the GISAXS signal comes from within the film as well as from the surface itself. In our case, the penetration depth is larger than the film thickness. A very smooth surface with few irregularities gives a GISAXS signal mostly confined to the specular plane, and this was ignored in our analysis, which was concentrated only on the particle scattering.

\section{Conclusion}

In conclusion, we have shown that the structure of GaAs nanoparticles obtained by nanosecond PLD on silicon substrate using $\mathrm{Ar}$ or $\mathrm{He}$ working gas can be tuned rather widely varying the deposition parameters, that is, working gas pressure and number of pulses. The sizes as well as the size distribution of the deposited nanoparticles are influenced by the number of pulses as was found also in vacuum [8], even before a compact film was formed. A low working gas pressure results in a relatively compact film with a wide variation in the size of the columnar structures in the film, while the use of higher pressure results in an increased roughness and more spherical particles. Our results suggest that He appears to be a more promising working gas than Ar for the better and smoother control of the GaAs nanoparticles growth by nanosecond PLD. In general, using $\mathrm{He}$, a narrower nanoparticle size distribution can be achieved for diverse pulse numbers and working gas pressure. This is most obvious from the formed particle height distribution under pressures lower than 1 mbar.

Keeping in mind the differences between the two methods we used to characterize the structure of the films, for example, the used wavelength of $0.154 \mathrm{~nm}$ for GISAXS as compared to the $\sim 15 \mathrm{~nm}$ tip radius of AFM and the probed area in GISAXS of about $50 \mathrm{~mm}^{2}$ while it is square micrometers in AFM, we can conclude that the correspondence of the obtained results is fair.

\section{Acknowledgments}

The financial support of the Ministry of Science, Education and Sports of the Republic of Croatia within the framework of the Projects 098-0982886-2866 and 035-0352851-2856 is gratefully acknowledged, as well as the help of M. Marcius and Dr. M. Ristic with SEM analysis.

\section{References}

[1] P. R. Willmott and J. R. Huber, "Pulsed laser vaporization and deposition," Reviews of Modern Physics, vol. 72, no. 1, pp. 315328,2000 .

[2] D. L. Smith, Thin-Film Deposition, Principles \& Practice, Mc Graw Hill, New York, NY, USA, 1995.

[3] P. Stampfli and K. H. Bennemann, "Time dependence of the laser-induced femtosecond lattice instability of Si and GaAs: role of longitudinal optical distortions," Physical Review B, vol. 49, no. 11, pp. 7299-7305, 1994.

[4] J. Bonse, K.-W. Brzezinka, and A. J. Meixner, "Modifying singlecrystalline silicon by femtosecond laser pulses: an analysis by micro Raman spectroscopy, scanning laser microscopy and atomic force microscopy," Applied Surface Science, vol. 221, no. 1-4, pp. 215-230, 2004.

[5] O. Varlamova, F. Costache, J. Reif, and M. Bestehorn, "Selforganized pattern formation upon femtosecond laser ablation by circularly polarized light," Applied Surface Science, vol. 252, no. 13, pp. 4702-4706, 2006.

[6] M. Henyk, F. Costache, and J. Reif, "Femtosecond laser ablation from sodium chloride and barium fluoride," Applied Surface Science, vol. 186, no. 1-4, pp. 381-384, 2002.

[7] Y. Lin, Q. Yu, R. Huang, W. Hang, J. He, and B. Huang, "Characterization of laser ablation and ionization in helium and argon: a comparative study by time-of-flight mass spectrometry," Spectrochimica Acta B, vol. 64, no. 11-12, pp. 1204-1211, 2009. 
[8] P. Dubček, B. Pivac, S. Milošević, N. Krstulović, Z. Kregar, and S. Bernstorff, "Pulsed laser ablation of GaAs using nano pulse length," Applied Surface Science, vol. 257, no. 12, pp. 5358-5361, 2011.

[9] J. Perrière, E. Millon, M. Chamarro, M. Morcrette, and C. Andreazza, "Formation of GaAs nanocrystals by laser ablation," Applied Physics Letters, vol. 78, no. 19, pp. 2949-2951, 2001.

[10] B. G. Streetman and B. Sanjay, Solid State Electronic Devices, Prentice Hall, New Jersey, NJ, USA, 5th edition, 2000.

[11] Y. Yoneda, "Anomalous surface reflection of X rays," Physical Review, vol. 131, no. 5, pp. 2010-2013, 1963.

[12] A. Erlacher, B. Ullrich, E. Y. Komarova, H. Jaeger, H. J. Haugan, and G. J. Brown, "Texture and surface analysis of thin-film GaAs on glass formed by pulsed-laser deposition," Journal of NonCrystalline Solids, vol. 352, no. 2, pp. 193-196, 2006. 

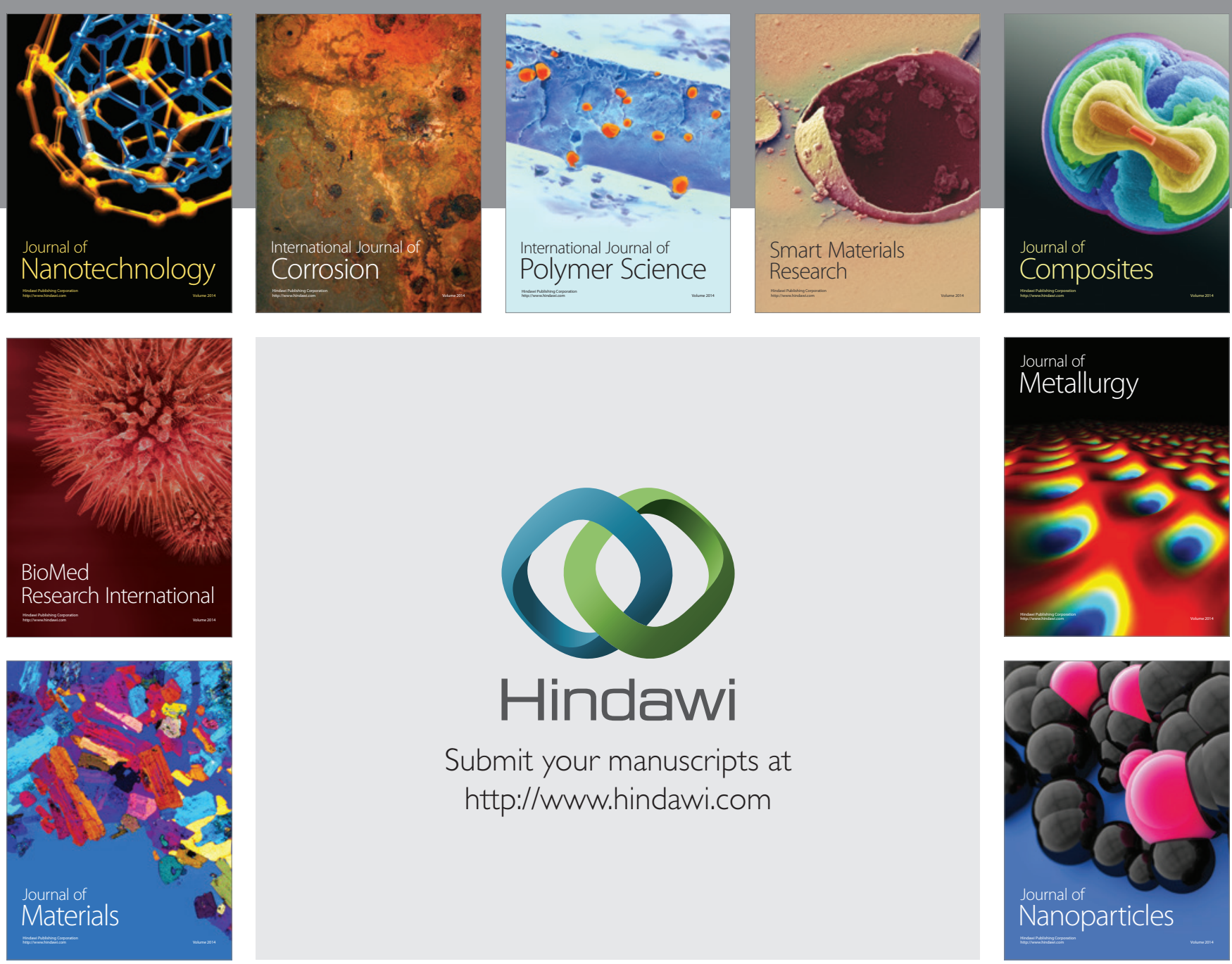

Submit your manuscripts at http://www.hindawi.com
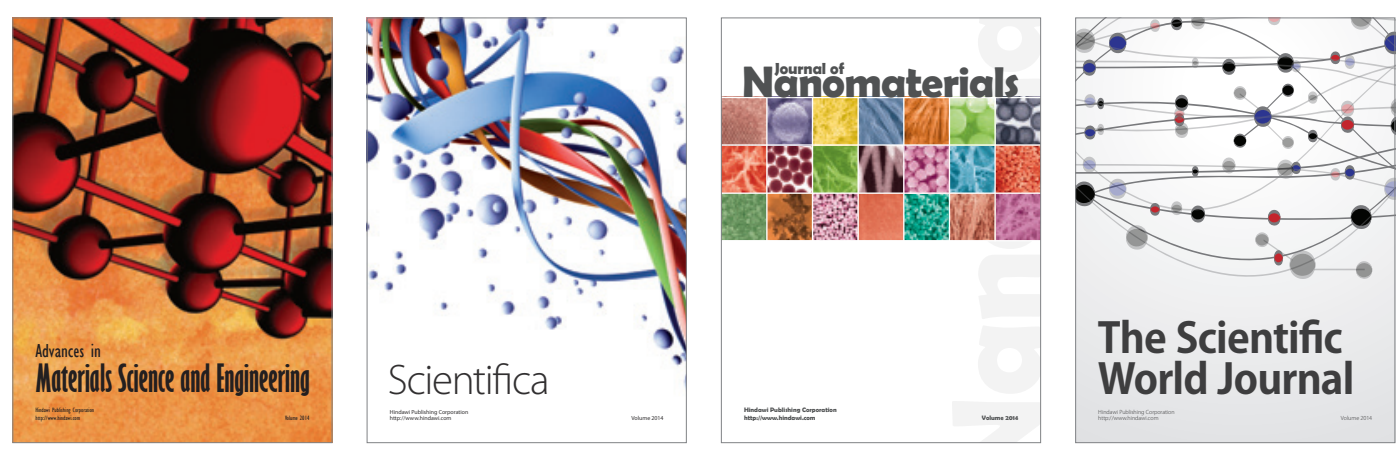

\section{The Scientific World Journal}
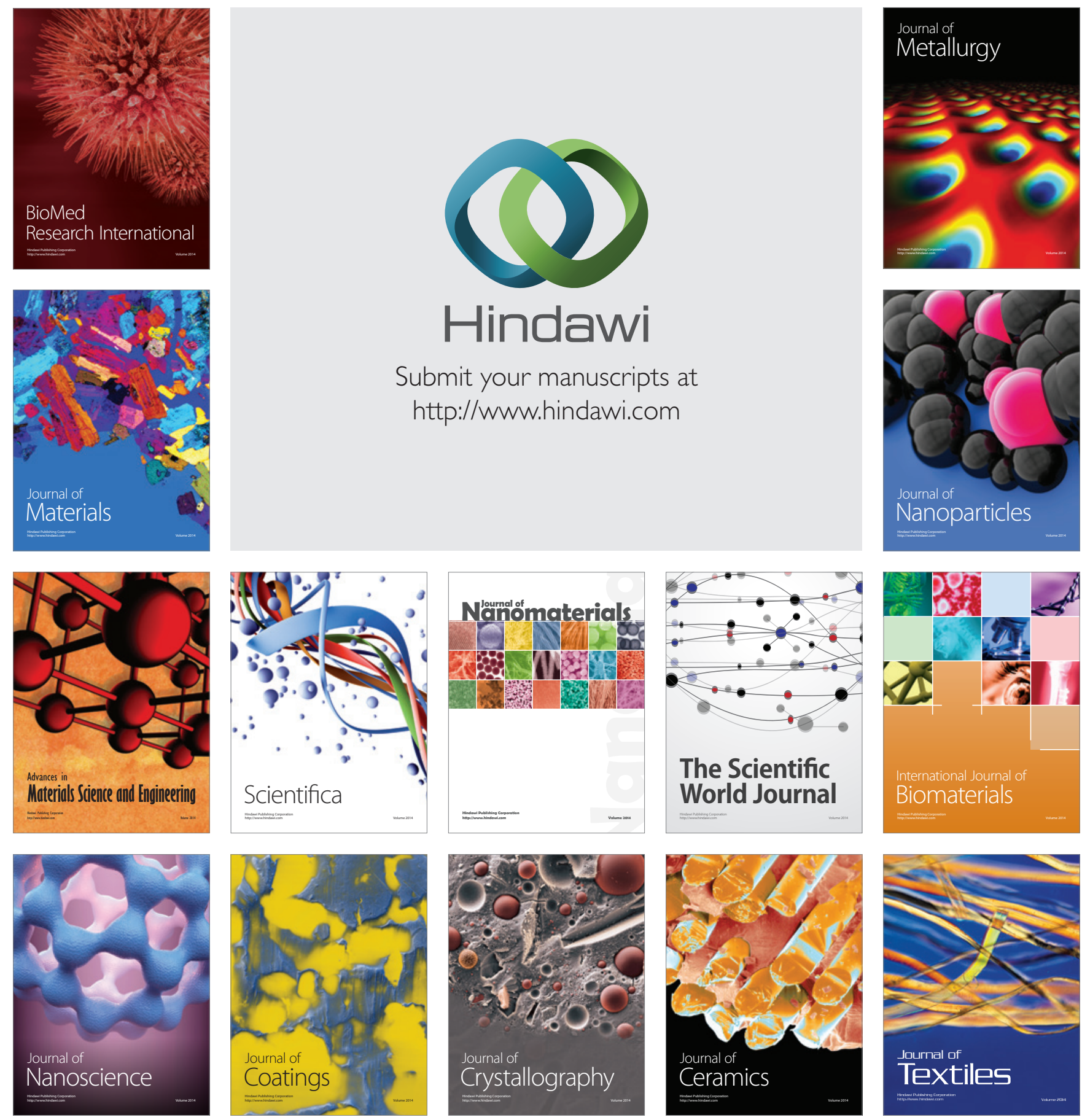